\title{
Non-Planar Geometrical Effects on the Magnetoelectrical Signal in a Three- Dimensional Nanomagnetic Circuit
}

\author{
Fanfan Meng, Claire Donnelly,* Claas Abert, Luka Skoric, Stuart Holmes, Zhuocong Xiao, \\ Jung-Wei Liao, Peter J. Newton, Crispin H.W. Barnes, Dédalo Sanz-Hernández, \\ Aurelio Hierro-Rodriguez, Dieter Suess, Russell P. Cowburn, and Amalio Fernández-Pacheco*
}

Cite This: ACS Nano 2021, 15, 6765-6773

Read Online

ACCESS | Llll Metrics \& More | 回 Article Recommendations | St Supporting Information

ABSTRACT: Expanding nanomagnetism and spintronics into three dimensions (3D) offers great opportunities for both fundamental and technological studies. However, probing the influence of complex 3D geometries on magnetoelectrical phenomena poses important experimental and theoretical challenges. In this work, we investigate the magnetoelectrical signals of a ferromagnetic $3 \mathrm{D}$ nanodevice integrated into a microelectronic circuit using direct-write nanofabrication. Due to the 3D vectorial nature of both electrical current and magnetization, a complex superposition of several magnetoelectrical effects takes place. By performing electrical measurements under the application of 3D magnetic fields, in

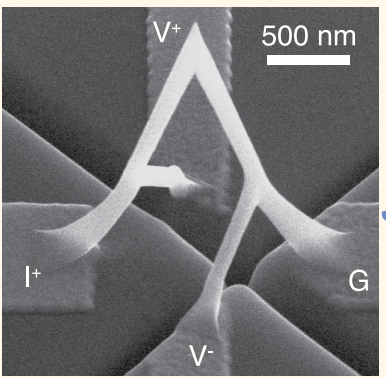

3D Nanomagnetic Circuit

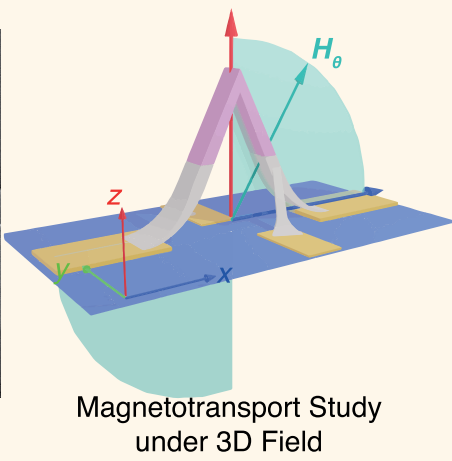
combination with macrospin simulations and finite element modeling, we disentangle the superimposed effects, finding how a 3D geometry leads to unusual angular dependences of wellknown magnetotransport effects such as the anomalous Hall effect. Crucially, our analysis also reveals a strong role of the noncollinear demagnetizing fields intrinsic to 3D nanostructures, which results in an angular dependent magnon magnetoresistance contributing strongly to the total magnetoelectrical signal. These findings are key to the understanding of 3D spintronic systems and underpin further fundamental and device-based studies.

KEYWORDS: magnetotransport, geometrical effects, 3D nanomagnetism, spintronics, 3D nanoprinting

S ince the discovery of the anisotropic magnetoresistance (AMR) by Lord Kelvin in $1857,{ }^{1}$ the fundamental investigation and exploitation of phenomena concerning the interplay between magnetism and electrical transport has seen incredible progress. ${ }^{2}$ Indeed, pioneering studies of intrinsic effects originating from spin-orbit coupling in ferromagnetic materials ${ }^{3}$ such as AMR and anomalous Hall effect $^{4}$ (AHE) have been followed by discoveries of the giant magnetoresistance $^{5}$ (GMR) and tunnel magnetoresistance ${ }^{6}$ (TMR). These effects have underpinned the magnetic data storage revolution of recent decades. ${ }^{2}$ Building upon this success, the field of spintronics in recent years has focused on control of spin states via electrical currents through the spintransfer torque ${ }^{7}$ (STT) effect, which has led to the recent development of nonvolatile random-access memory (MRAM) devices. ${ }^{8}$ All these advances, together with its role in today's digital world, make spintronics one of the most successful areas of nanotechnology. ${ }^{2}$ Today, alternative forms of controlling the magnetic state via different mechanisms, e.g., spin-orbit torques $^{9}$ (SOT), electric fields, ${ }^{10}$ and optical probes ${ }^{11}$ are garnering much interest, with the prospect that future spintronic devices will impact a significant number of technological areas, ${ }^{8}$ including the emerging field of neuromorphic computing. ${ }^{12}$

To meet the ever-increasing demands for high functionality and more energy efficient devices, fundamental paradigm shifts are required. One of the most promising innovations involves the expansion of spintronics into three dimensions $(3 \mathrm{D})^{13}$ which, with advantages such as higher density and enhanced

Received: December 8, 2020

Accepted: March 30, 2021

Published: April 13, 2021 


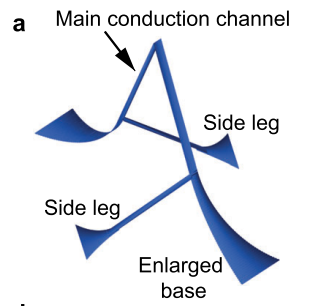

d

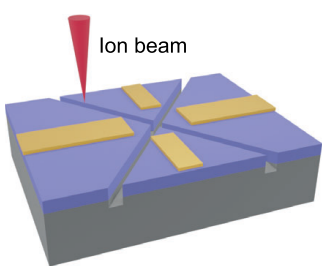

b

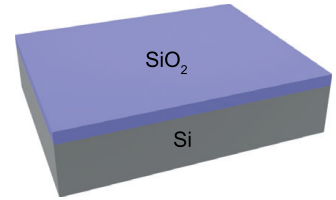

e

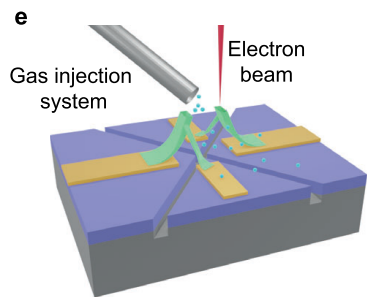

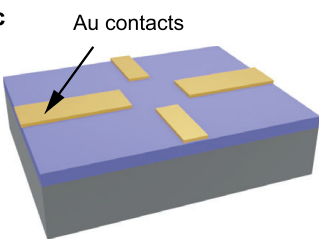

f

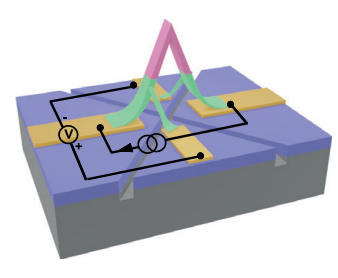

g

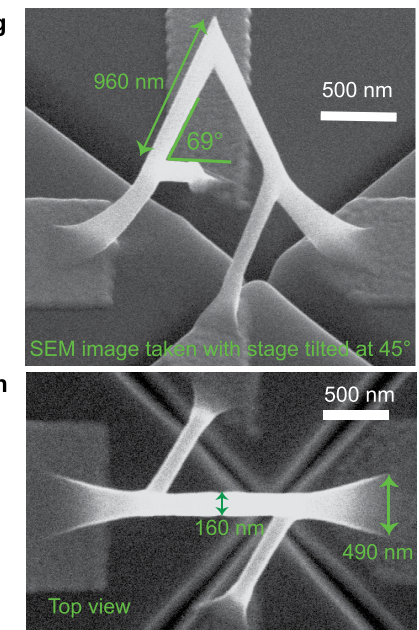

Figure 1. Integration of a ferromagnetic $3 \mathrm{D}$ nanobridge in a microelectronic circuit. a, $\mathrm{A}$ rendering of the $\mathrm{CAD}$ design of the nanobridge investigated in the experiment. $b-e$, Steps followed to fabricate the nanobridge: $b$, preparation of a clean silicon dioxide substrate. c, patterning of electrical contacts by electron-beam lithography and electron-beam evaporation. $d$, milling of trenches by focused ion beam. e, 3D-nanoprinting of the bridge via focused electron beam induced deposition. $f$, Four-probe magnetotransport measurement configuration, where the voltage across the pink region is measured. $\mathrm{g}$, SEM image of the side view of the as fabricated nanomagnetic circuit. Image tilt $45^{\circ}$. $h$, SEM image of the top view of the fabricated nanomagnetic circuit.

device connectivity, offers a wealth of opportunities for 3D spintronics devices. With proposals ranging from the $3 \mathrm{D}$ magnetic racetrack memory ${ }^{14}$ and the magnetic rachet $^{15}$ that represent alternative routes to ultrahigh density, high-performance logic and memory devices, to $3 \mathrm{D}$ interconnected memristors for neuromorphic computing, ${ }^{12,16,17} 3 \mathrm{D}$ spintronics offers a highly efficient answer to the demands the field is currently facing. Experimentally, there has been a recent surge in progress including the observation of fast domain wall velocities in cylindrical nanowires, ${ }^{18}$ the demonstration of field-mediated controllable domain wall movement in 3D conduits ${ }^{19}$ and extensive degeneracy ${ }^{20}$ in frustrated nanowire lattices. $^{21}$ The higher degrees of freedom and surface-tovolume ratio of $3 \mathrm{D}$ nanostructures also make them desirable for sensing applications. ${ }^{13}$ In this realm, there have already been demonstrations, including magnetic imaging with highaspect-ratio nanowires used as high-resolution MFM tips ${ }^{22}$ and flexible position sensors making use of 3D nanomembranes. ${ }^{23}$

As well as offering exciting prospects for devices, the introduction of 3D geometrical effects (e.g., curvature and chirality) also provide opportunities for exciting physics. ${ }^{13,24}$ These include predictions for complex magnetic textures ${ }^{25,26}$ and curvature-induced effects, ${ }^{27-30}$ as well as exotic dynamic behavior. ${ }^{18,31,32}$ Although the experimental realization of such effects is challenging, recent developments in synthesis ${ }^{21,33-36}$ and characterization techniques ${ }^{26,37-42}$ have led to a number of stimulating confirmations and discoveries of the potential of 3D nanomagnetism. ${ }^{13,24,43}$ For example, nonreciprocal spinwave propagation ${ }^{31,44}$ has been observed in rolled-up nanomembranes, while magnetic chiral spin textures have been realized in double helices. ${ }^{45}$ When combined, the fields of 3D spintronics and nanomagnetism offer great potential for functional devices.

Before it becomes possible to fully exploit the potential of 3D spintronics, however, a fundamental understanding of the influence of the $3 \mathrm{D}$ geometry on the magnetotransport properties is needed. In this paper, we demonstrate the direct integration of a complex 3D magnetic nanostructure into a microelectronic circuit via direct-write nanoprinting and characterize the behavior of intrinsic magnetotransport effects such as the AMR and AHE in a 3D nanocircuit under the application of external magnetic fields. The efficient integration of the $3 \mathrm{D}$ magnetic structure, together with the vectorial nature of both current and magnetization in $3 \mathrm{D}$ nanostructures, results in an unconventional superposition of different magnetotransport effects that are measured simultaneously. We separate these different contributions by taking advantage of symmetry arguments and their distinct angular dependence in response to magnetic fields. This allows us to understand the unexpected angular dependence of AHE due to the 3D geometry, and crucially reveal the strong influence of the noncollinear demagnetizing field on the $3 \mathrm{D}$ magnetotransport via the magnon magnetoresistance (MMR). These fundamental insights are key for the future study of spintronic effects in $3 \mathrm{D}$ magnetic nanostructures, as well as the realization of $3 \mathrm{D}$ spintronic technologies.

\section{RESULTS AND DISCUSSION}

Fabrication of 3D Nanomagnetic Circuits. One of the key building blocks in $3 \mathrm{D}$ spintronics is the magnetic nanobridge. This device not only interconnects electrical and magnetic parts in the nanomagnetic circuit, ${ }^{46}$ but can also host magnetic domain walls (DWs) ${ }^{47}$ and magnetic spin waves, ${ }^{48}$ to serve as both a memory element ${ }^{14}$ and a logic gate, ${ }^{49}$ offering the possibility of nontraditional computing architectures in which the boundaries between interconnects, memory and logic are eliminated. ${ }^{13} \mathrm{~A}$ rendering of the bridge design investigated in this study is shown in Figure 1a where, in additional to the main conduction channel, two side-legs are introduced to allow standard four-probe measurements. These side legs are placed diagonally across the main channel so that both longitudinal and transverse magnetoelectrical signals can be measured simultaneously, therefore providing complementary information about the magnetic state of the device (as discussed later in the Magnetotransport Measurements section). This arrangement promotes an efficient use of space on the substrate as only four planar pads are required to fully probe a high aspect ratio $3 \mathrm{D}$ circuit. It also improves the 

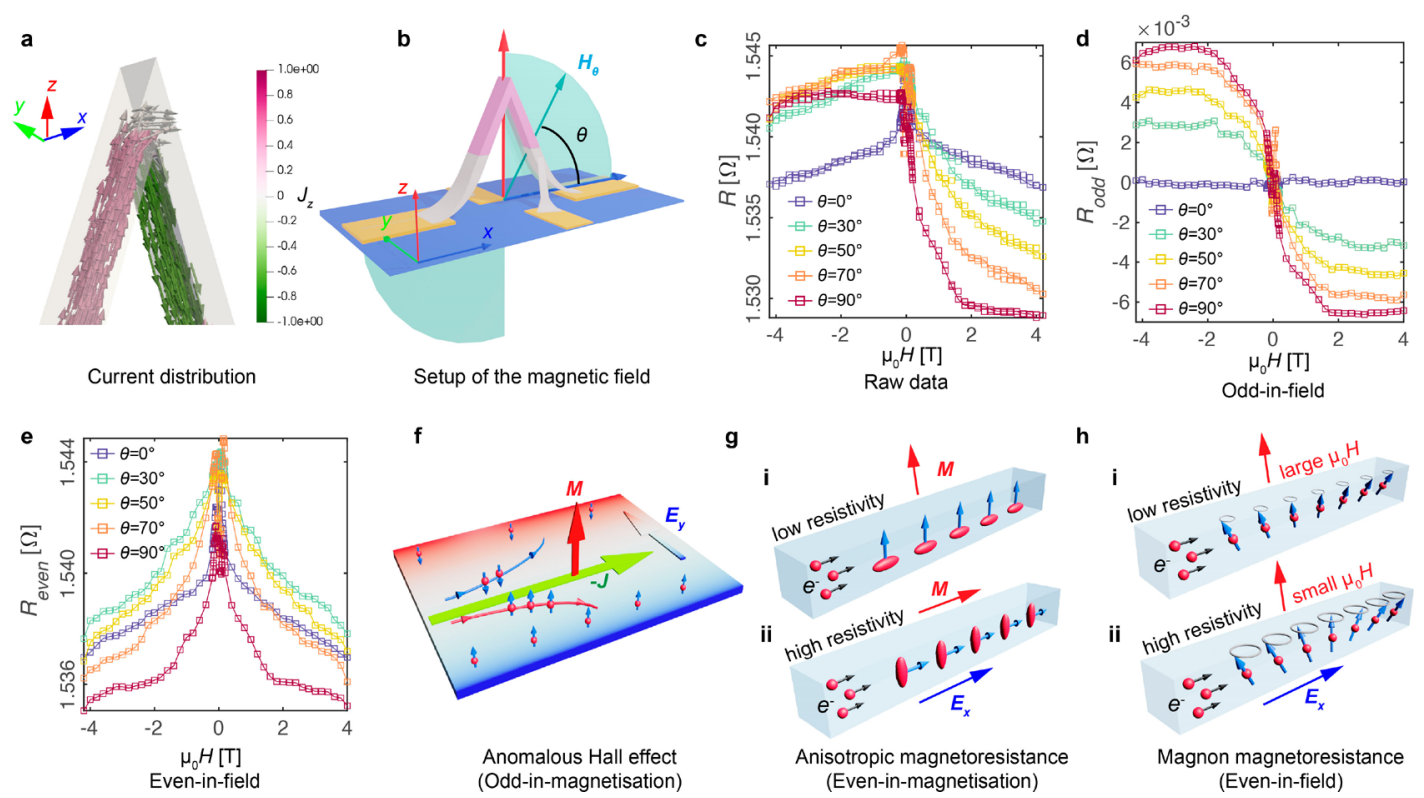

Figure 2. Magnetotransport measurements. a, The simulation of the current density in the probed region of the 3D nanobridge with the color indicating the $z$ component of the current density. $b$, The schematic shows how the field is applied relative to the 3D nanobridge, $\theta$ is the angle between the applied field and the substrate. c, MT hysteresis loops obtained from -4 to 4 and $4 \mathrm{~T}$ to $-4 \mathrm{~T}$ for each field angle $\theta$ from $0^{\circ}$ to $90^{\circ}$. d, The odd-in-field signal, $R_{\text {odd }}$ e, The even-in-field signal, $R_{\text {even }}$. $\mathrm{f}-\mathrm{h}$, Schematics showing the main magnetotransport effects considered in the model: $f$, anomalous Hall effect: electrons with opposite spins are deflected in different directions due to the spin-orbit coupling mediated intrinsic and extrinsic (skew-scattering and side-jump) scattering-related mechanisms. ${ }^{4}$ g, anisotropic magnetoresistance: variation in resistance induced by different degrees of scattering of spin-orbit coupled carriers. ${ }^{57} \mathrm{~h}$, magnon magnetoresistance: reduced resistance due to the suppression of spin waves by an applied magnetic field. ${ }^{58}$

mechanical stability ${ }^{50}$ of the device, by having rotationalsymmetric leads at both sides of the main bridge. The enlarged bases of the bridge used here are designed to improve the electrical contact with the pads.

The realization of a 3D nanomagnetic circuit faces two challenges. The first challenge consists of creating an arbitrary $3 \mathrm{D}$ geometry with the desired material properties. The second challenge involves the integration of $3 \mathrm{D}$ magnetic structures into 2D microelectronics circuits. To overcome these issues, we employ focused electron beam induced deposition (FEBID), an additive fabrication technique with a spatial resolution of 10 s of nanometers. ${ }^{50-52}$ Inspired by conventional $3 \mathrm{D}$ printers, recent developments in FEBID now make it possible to design beam scanning instructions of almost arbitrary 3D nanostructure geometries with varying curvatures and topologies, directly from standard 3D computer aided design (CAD) files. ${ }^{53}$ In this way, with the appropriate use of precursor gases, 3D structures composed of high-quality ferromagnetic materials ${ }^{54,55}$ can be fabricated directly on almost any substrate. ${ }^{50}$ These capabilities make FEBID an ideal technique for the integration of a $3 \mathrm{D}$ magnetic circuit onto prepatterned electrical contacts.

To begin the fabrication process of the $3 \mathrm{D}$ nanomagnetic circuit, four $50 \mathrm{~nm}$ thick gold contacts were patterned and deposited on a silicon substrate with a $300 \mathrm{~nm}$ thick silicon dioxide layer via electron beam lithography and electron beam evaporation (Figure 1b,c). Prior to FEBID 3D printing, trenches were milled by $\mathrm{Xe}^{+}$focused ion beam (FIB) between contacts to minimize the influence of conducting parasitic deposits, $^{50}$ which could interfere during the transport measurements of the bridge (Figure 1d). Next, the nanobridge was directly printed on the four contacts via FEBID (Figure 1e) using dicobalt octacarbonyl $\left(\mathrm{Co}_{2}(\mathrm{CO})_{8}\right)$ as a precursor, under conditions which have been shown to result in greater than 95 at. \% cobalt with nanocrystalline microstructure. ${ }^{54,55}$ During the measurement, an AC current with a constant peak to peak value of $0.6 \mu \mathrm{A}$ is supplied through the main leg of the bridge while the voltage is measured across the side leg contacts, as shown schematically in Figure 1f. Scanning electron microscopy (SEM) images of the resulting nanomagnetic circuit are shown in Figure 1g,h, demonstrating the successful connection of this complex, high aspect ratio 3D nanostructure to a planar circuit patterned on a substrate using well-defined leads. The legs of the probed region of the bridge are $960 \pm 40 \mathrm{~nm}$ long and $160 \pm 5 \mathrm{~nm}$ wide, forming an angle of $68.6 \pm 0.8^{\circ}$ with the substrate. A small asymmetry in the bridge is observed, corresponding to a difference in the angles formed by the two legs and the substrate of $1.6 \pm 0.8^{\circ}$. Detailed dimensions of the printed bridge and further information about the device are given in the Supporting Information (SI).

Magnetotransport Measurements. Following the realization of the 3D nanomagnetic circuit, we next consider magnetotransport (MT) measurements of the 3D nanostructure. The measurement setup used (Figure 1f) provides access to magnetoelectrical signals with different symmetries and, due to the 3D profile of the current (Figure 2a), a superposition of different MT effects was measured. To understand the contribution of different MT effects to the total signal probed in the device, and the influence of the $3 \mathrm{D}$ geometry, we performed measurements with the sample at different orientations with respect to the applied magnetic field direction. In this way, we can exploit the angular dependence and symmetries to separate the different MT effects. ${ }^{56}$

To probe the $3 \mathrm{D}$ response of the transport properties, we measure a full MT hysteresis loop from -4 to $4 \mathrm{~T}$ and from 4 
$\mathrm{T}$ to $-4 \mathrm{~T}$ at $180 \mathrm{~K}$, for fields applied between $\theta=0^{\circ}$ and $\theta=$ $90^{\circ}$, with an interval of $10^{\circ}$ in the $X Z$ plane (Figure $2 \mathrm{~b}$ ), plotted from violet to red in Figure $2 c$. The raw data (a selection of angles shown in Figure 2c) shows a clear angular dependence, with the signal becoming less symmetric as the angle changes from $\theta=0^{\circ}$ to $\theta=90^{\circ}$. In 2D, Hall bars are often patterned to separate various MT effects. Here, we instead make use of the different symmetries of the signal with respect to the sense of the applied field, to separate coexisting MT effects. Specifically, the raw data is first separated into the odd-in-field part as $R_{\text {odd }}=(R(\mathrm{H})-R(-\mathrm{H})) / 2$, and the evenin-field part as $R_{\text {even }}=(R(\mathrm{H})+R(-\mathrm{H})) / 2$ as shown in Figure $2 \mathrm{~d}$,e, respectively. In this study, we focused on the high field range only, where the magnetization is fully reversible, so that odd- and even-in-field signals correspond to odd- and even-inmagnetization effects.

With the raw data separated into odd and even parts, we compare them to the symmetries and angular dependences of the anisotropic magnetoresistance (AMR), planar Hall effect (PHE), anomalous Hall effect (AHE) and ordinary Hall effect (OHE) on the current, internal magnetization and magnetic field induction: ${ }^{3}$

$$
\boldsymbol{E}=\rho_{\perp} \boldsymbol{J}+\underbrace{(\overbrace{\rho_{\|}-\rho_{\perp}}^{\rho_{G}}[\boldsymbol{m} \cdot \boldsymbol{J}] \boldsymbol{m}}_{\text {AMR }+ \text { PHE }}+\underbrace{\rho_{A H E} \boldsymbol{m} \times \boldsymbol{J}}_{\text {AHE }}+\underbrace{R_{O H E} \boldsymbol{B} \times \boldsymbol{J}}_{\text {OHE }}
$$

where $E$ is the electric field, $J$ is the current density vector, $\boldsymbol{m}$ is a unit vector in the magnetization direction, $\rho_{\|}$is the resistivity for $J$ parallel to $m, \rho_{\perp}$ is the resistivity for $J$ perpendicular to $m$, $\rho_{\mathrm{AHE}}$ is the anomalous Hall resistivity, $R_{\mathrm{OHE}}$ is the ordinary Hall coefficient $\left(R_{\mathrm{OHE}}=\rho_{\mathrm{OHE}} / B\right.$, where $\rho_{\mathrm{OHE}}$ is the ordinary Hall resistivity, which is a function of $B$ ) and $B$ is the total magnetic field induction, $\boldsymbol{B}=\mu_{0}\left(\boldsymbol{H}_{\mathrm{a}}+\boldsymbol{H}_{\mathrm{d}}+\boldsymbol{M}\right)$. Here, $\boldsymbol{H}_{\mathrm{a}}$ is the applied field, $\boldsymbol{H}_{\mathrm{d}}$ is the demagnetizing field and $\boldsymbol{M}$ is the magnetization.

As the AHE is an odd-in-magnetization effect (Figure 2f), the induced transverse electric field changes in sign with the reversal of magnetization, and its strength depends on the component of magnetization perpendicular to the current $\left(m_{\perp}\right){ }^{3}$ The ordinary Hall effect is also an odd-in-field effect, and is usually a much smaller effect compared to the AHE. ${ }^{3}$ The odd signal is plotted in Figure 2d, where first, we observe that for all $\theta$ values, $R_{\text {odd }}$ appears to level off for applied fields greater than $2 \mathrm{~T}$. As the AHE dominates the odd signal and depends on the magnetization only, this indicates that the magnetization is effectively saturated at a field around $2 \mathrm{~T}$. Above $2 \mathrm{~T}$, a small negative slope can be observed (most significant at $\theta=90^{\circ}$ ), which we attribute to the ordinary Hall effect $^{54}$ (see below for more details).

We next consider the even-in-magnetization effects, the AMR (Figure 2g) and PHE, which are the longitudinal and transverse components of the anisotropic resistivity and remain the same when the magnetization reverses, with their magnitude depending on the magnetization parallel to the current direction $\left(m_{\|}\right), 3,56,57,59$ as given by eq 1 . The even signal measured in the bridge is plotted in Figure 2e, where we notice that for all $\theta$, the resistance is always the highest when the applied field is around $0 \mathrm{~T}$. This can be understood as the magnetization at remanence tends to align along the long (easy) axes of the bridge due to shape anisotropy, which coincides with the current direction for this geometry. Since cobalt has a positive AMR ratio, ${ }^{54}$ the resistance is highest when the magnetization and current directions are aligned. Second, in contrast to the odd signal, the even signal does not saturate at fields above $2 \mathrm{~T}$ but instead decreases further with applied field. As $\left(\rho_{\|}-\rho_{\perp}\right)$, that is, the AMR term in eq 1 , is not expected to change significantly after saturation, ${ }^{60}$ we attribute the measured change to the magnon magnetoresistance (MMR), ${ }^{58,61}$ which has been reported as a linear and nonsaturating negative MR present after all magnetic moments are fully saturated. As schematically shown in Figure $2 \mathrm{~h}$, this contribution is due to the progressive suppression of spin disorder caused by spin waves in a ferromagnet under an increasing field strength, which results in a drop of resistance due to a reduction in the electron-magnon scattering. ${ }^{58,61,62}$ The magnitude of MMR depends on the strength, and not the sign of the applied field, and has therefore an even response to the applied field.

Magnetotransport Effects at High Fields. To obtain a quantitative understanding of the different contributions of the mentioned effects, we study the angular dependence of $R_{\text {odd }}$ and $R_{\text {even }}$ at high fields $( \pm 4 \mathrm{~T})$. We focus on the magnetoelectrical signals at high fields, where the magnetic state is close to uniform, and where the angular dependence of MT signals is usually the fingerprint of the underlying physical mechanisms. ${ }^{2}$ In order to understand our measurements, we take into account the $3 \mathrm{D}$ nature of both the magnetization and the current distribution, by making use of both a multimacrospin model and a finite element method (FEM) analysis, as explained below.

First, we determine the magnetic state of the nanobridge at $\pm 4 \mathrm{~T}$ for different magnetic field directions, by modeling the magnetic configuration using an adapted multimacrospin (multiple single domain) approximation: the probed region of the structure is considered to be made up of three singledomain sections, as marked in green, pink and yellow, respectively in Figure 3a. The interaction between the three regions is not considered in the model, that is, the magnetization vectors for each section, $m_{1}(\theta), m_{2}(\theta)$ and $\boldsymbol{m}_{3}(\boldsymbol{\theta})$ are determined independently, by minimizing the Zeeman and magnetostatic energies of each section at a given $\boldsymbol{\theta}$, where the shape anisotropy of each part is included as an independent demagnetizing term. Due to the nanocrystalline nature of FEBID Co under these growth conditions, ${ }^{54}$ which results in magnetic properties dominated by shape anisotropy, we do not consider the intrinsic magnetocrystalline anisotropy of cobalt in the model. As described below, this approach is sufficient to fully understand the magnetic behavior of the nanocircuit from MT signals under the application of high magnetic fields. The detailed calculation is described in the SI.

After obtaining the magnetic configuration from the macrospin model, we simulate the MT signal by solving the electric potential $u$ across the side contacts using a finite element method (FEM) with a CAD-based FEM mesh that reproduces the dimensions of the printed nanobridge (Figure 3a). The influence of different MT effects is summarized as a magnetization- and field- dependent resistivity tensor $\rho(m, B)$ which can be obtained from eq 1 (see Methods section). For each section and angle $\theta$ considered, a different resistivity tensor is calculated from the modeled magnetization distribution $\boldsymbol{m}(\theta)$ and total field distribution $\boldsymbol{B}(\theta)$, and assigned to the corresponding sections of the nanobridge. We note that a nonmagnetic conducting layer underneath the bridge due to known parasitic deposition of cobalt ${ }^{63}$ has been included in the simulations for a better quantitative agreement of the base resistance. Details of the FEM simulation setup are 
a

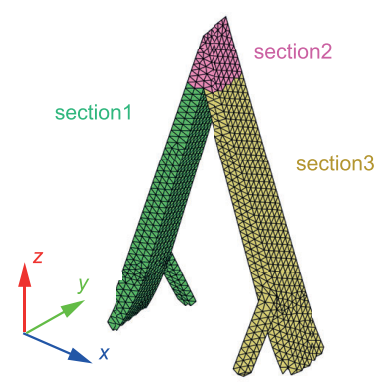

FEM mesh

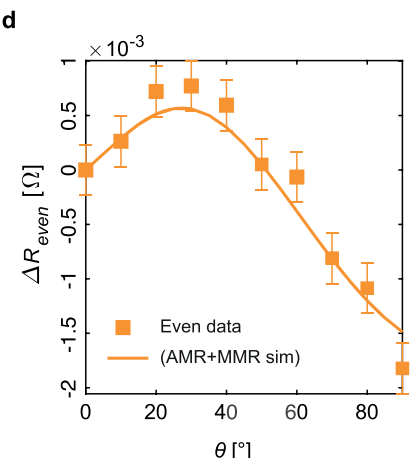

Even part: $A M R+M M R$

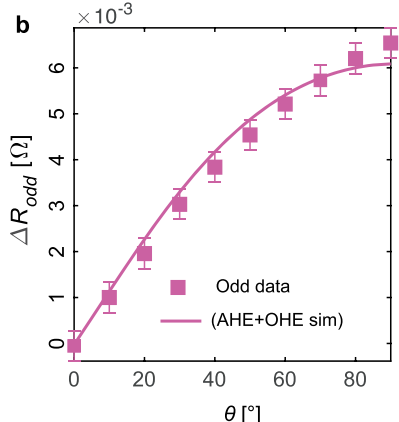

Odd part: $\mathrm{AHE}+\mathrm{OHE}$

e

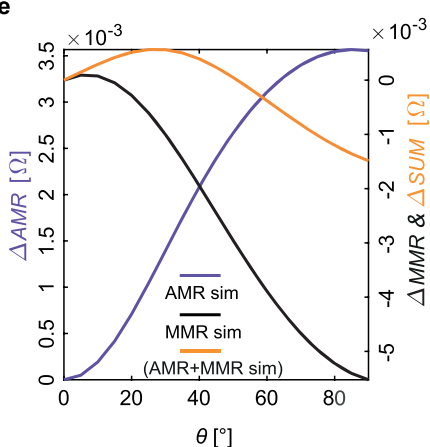

Even part: AMR+MMR

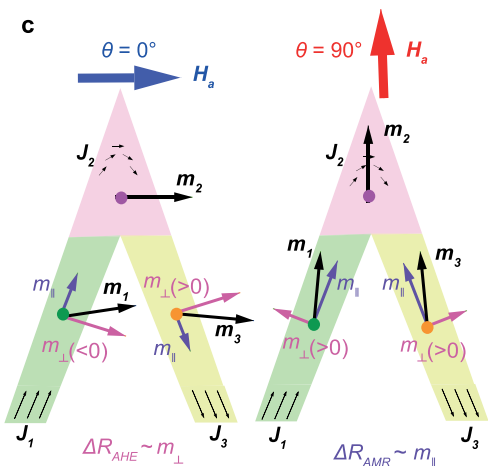

f

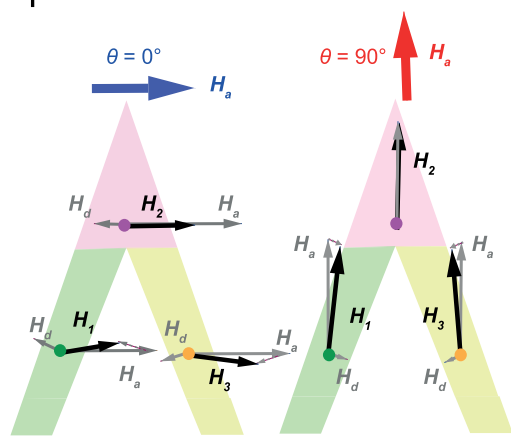

$\Delta R_{M M R} \sim|H|$

Figure 3. Analysis of resistance data at 4 T. a, The FEM mesh of the bridge is divided into three domains. b, Comparison between the angular dependence of the odd part data and the AHE and OHE simulation. c, Averaged magnetization vectors, $m_{1}(\theta)$, $m_{2}(\theta)$, and $m_{3}(\theta)$ for the three sections for $\theta=0^{\circ}$ and $\theta=90^{\circ}$ and their components on the current and current normal directions. $d$, Comparison between the angular dependence of the even part of the data and the sum of AMR and MMR simulation. e, Simulation of the AMR, MMR effects and their sum. f, Applied field $H_{\mathrm{a}}$, demagnetizing field $H_{\mathrm{d}}$ and their vector sum $H$ for each section of the bridge.

given in the SI. After obtaining the potential difference between side contacts for fields applied in all directions, the odd part and even part of the simulated results can be separated in the same way as for the experimental data, for comparison.

We first consider the angular dependence of the odd signal by plotting the average of $\left|R_{\text {odd }}(4 \mathrm{~T})\right|$ and $\left|R_{\text {odd }}(-4 \mathrm{~T})\right|$ with respect to the $\theta=0^{\circ}$ case (squares in Figure 3b), along with the simulated odd signal (line in Figure $3 \mathrm{~b}$ ), which is dominated by the AHE (see SI). We observe a continuous increase in the magnitude of the odd component as the field rotates from $\theta=0^{\circ}$ to $\theta=90^{\circ}$, and a good agreement between the simulations and the data, confirming that the odd signal is due to a combination of the AHE and the smaller OHE. ${ }^{54} \mathrm{We}$ first consider the dominant contribution of the AHE. The trend of an increasing AHE magnitude with increasing $\theta$ can be understood intuitively by considering the $\theta=0^{\circ}$ and $\theta=90^{\circ}$ extreme cases in Figure 3c, where the magnetization vector $m$ calculated for each section from the macrospin model is shown. The component of the magnetization on the current direction ( $m_{\|}$, purple) and on the current-normal direction $\left(m_{\perp}\right.$, pink) are also plotted. As the AHE depends on $m_{\perp}$, at a first glance it might appear that the $\theta=0^{\circ}$ case would result in a larger AHE effect, as the magnitude of $m_{\perp}$ is larger for the green and yellow sections. However, the geometry of the nanobridge results in opposite signs of $m_{\perp}$ for these two sections in the frame of reference of the current, and thus the two AHE signals cancel out. Using analogous arguments for the pink region, the current turns its direction along this section, also resulting in a negligible AHE signal. The opposite scenario occurs at $\theta=90^{\circ}$, where the same sign of $m_{\perp}$ for all three sections leads to the signals adding up. The $3 \mathrm{D}$ current distribution and contact arrangement lead to the AHE being sensitive to multiple components of the magnetization, both $\mathrm{m}_{x}$ and $\mathrm{m}_{z}$, and thus making it possible for the AHE to cancel, as is observed at $\theta=0^{\circ}$. This unusual effect constitutes a demonstration of how vectorial current distributions flowing in 3D magnetic geometries may lead to deviations of the angular dependence of MT signals from familiar patterns observed in planar systems. The magnitude of OHE follows the same trend, which explains why the negative linear slope is the most obvious at $\theta=90^{\circ}$ in Figure 2d. From this analysis, we also obtain the AHE and OHE resistivities of the device. From the model fitting, we find $\rho_{\mathrm{AHE}}=5.6 \times 10^{-9} \Omega \mathrm{m}$ and $\rho_{\mathrm{OHE}}=-1.2$ $\times 10^{-10} \Omega \mathrm{m} / \mathrm{T}$, of the same range as the anomalous Hall resistivity and ordinary Hall coefficient reported in the literature for FEBID-deposited cobalt. ${ }^{54}$

We next consider the even part of the signal, with the average of $R_{\text {even }}(4 \mathrm{~T})$ and $R_{\text {even }}(-4 \mathrm{~T})$ with respect to the $\theta=$ $0^{\circ}$ case, plotted as squares in Figure $3 \mathrm{~d}$. We observe a peak at around $\theta=30^{\circ}$, as well as an increased resistance at $\theta=0^{\circ}$ compared to $\theta=90^{\circ}$. We first examine what are commonly considered the main intrinsic contributions to the even signal: the AMR and PHE. The simulated sum of AMR and PHE signals (referred for brevity as AMR) is plotted in Figure 3e (purple line), which takes the form of a monotonic increase in the resistance with increasing $\theta$. Again, this angular dependence can be understood by considering $m_{\|}$at $\theta=0^{\circ}$ and $\theta=$ $90^{\circ}$ in Figure $3 \mathrm{c}: m_{\|}$is larger at $\theta=90^{\circ}$, so we would expect the resistance to be larger at $\theta=90^{\circ}$ due to AMR and PHE, 
consistent with the simulated signal. However, the experimental data in Figure $3 \mathrm{~d}$ exhibit a very different angular dependence, implying that the even data cannot be fully explained by these two effects.

To understand the significant difference in the even part of the signal, we consider the angular dependence of magnon magnetoresistance as an additional contribution. The MMR results in a change of resistivity that can be described by the electron-magnon scattering model developed by Raquet et al.: $58,61,64$

$$
\Delta \rho_{\mathrm{mmr}}(T, B) \approx \rho(T, B)-\rho(T, 0) \propto \frac{B T}{D(T)^{2}} \ln \left(\frac{\mu_{\text {Bohr }} B}{k_{\mathrm{B}} T}\right)
$$

where $T$ is the temperature, $D(T)$ is the temperature dependent magnon stiffness, $\mu_{\text {Bohr }}$ is the Bohr magneton, $k_{B}$ is the Boltzmann constant, and $B$ is the projection of the total effective magnetic field, $\boldsymbol{B}=\mu_{0}(\boldsymbol{H}+\boldsymbol{M})$, on the direction of the magnetization, that is, the magnitude of the total effective field, acting to suppress the magnitude of spin-waves present in the system. Here $\boldsymbol{H}$ is the vector sum of the applied field $\boldsymbol{H}_{\boldsymbol{a}}$ and demagnetizing field $\boldsymbol{H}_{a}, \boldsymbol{H}=\boldsymbol{H}_{\boldsymbol{a}}+\boldsymbol{H}_{d}$, and $\boldsymbol{M}$ is the magnetization.

For a constant temperature, Equation 2 leads to a negative change of resistivity that decreases almost linearly with the magnitude of the effective field, which is consistent with the even part of the experimental signal for applied fields greater than $2 \mathrm{~T}$ (Figure $2 \mathrm{e}$ ). Although $\Delta \rho_{\mathrm{mmr}}$ is not dependent on the direction of the magnetization with respect to the current, ${ }^{58,61}$ this does not necessarily mean that no change in the $\Delta \rho_{\mathrm{mmr}}$ will result from different directions of the applied magnetic field. Previous studies investigating the contribution of MMR in nanostructures have mainly focused on measurements under fields applied along the easy axis of $2 \mathrm{D}$ thin films or nanowires, ${ }^{58,61}$ where the demagnetizing field is negligible, leading to an effective field equivalent to the applied field. ${ }^{58,61}$ However, in the case of a $3 \mathrm{D}$ nanocircuit such as the one studied here, the nonplanar geometry results in an applied field always oblique to at least one section of the circuit. This results in a nonzero demagnetizing field modifying the effective magnetic field at any angle.

In order to compare the experimental MMR with simulations, we calculate the nonzero demagnetizing field as a function of $\theta$ from the macrospin model, as described in the SI. The resulting change of resistivity, $\Delta \rho_{\text {mmr }}$, plotted as a black line in Figure $3 \mathrm{e}$, is obtained from eq 2 . An opposite trend in the angular dependence of MMR with respect to the AMR is observed, with the resistance becoming more negative with increasing angle. Again, we consider the extreme cases of $\theta=$ $0^{\circ}$ and $\theta=90^{\circ}$ in Figure $3 \mathrm{f}$ to understand this angular dependence intuitively. At $\theta=0^{\circ}$, the applied field has a larger component perpendicular to each section's easy axis, resulting in a larger demagnetizing field, and thus a lower magnitude of the overall effective magnetic field, leading to a higher resistance. At $\theta=90^{\circ}$, the field is more aligned with the easy axes for all three sections, leading to a lower demagnetizing field, and a larger magnitude of the total effective field, associated with a larger drop of resistivity, as seen in the simulated data.

Finally, we compare the sum of AMR, PHE and MMR obtained from simulations (Figure $3 \mathrm{~d}$,e, orange line), to the even data (Figure 3d, squares). An excellent agreement with experiments is observed, with the maximum resistance at around $\theta=30^{\circ}$, and the overall angular trend well reproduced. This demonstration of the strong influence of the threedimensional geometry on the magnetotransport reveals the importance of noncollinear alignments between magnetic fields and geometry in nonplanar magnetic nanocircuits. In particular, this work demonstrates how magnetostatic interactions in 3D geometries manifest through a significant deviation of the MMR contribution.

\section{CONCLUSIONS}

We have investigated the magnetoelectrical response of a 3D nanomagnetic circuit created by advanced 3D nanoprinting. By exploiting signal symmetries with respect to magnetic field configurations, we were able to address the superposition of different magnetotransport effects. Specifically, we combined electrical measurements with finite-element calculations to disentangle and understand key magnetotransport effects (Hall effect and magnetoresistance signals) within the nanocircuit, obtaining a clear understanding of their magnitudes and angular dependences as a function of external magnetic fields applied along multiple directions. In this way, we identified that the $3 \mathrm{D}$ geometry of the magnetic nanostructure has a major effect, inducing deviations of the Hall effect signal from the angular dependence usually observed in planar geometries. The $3 \mathrm{D}$ vector nature of both the magnetization and current is responsible for this unusual angle dependence, due to the fact that signals that, for example, cancel out in planar geometries may add up in 3D. Moreover, the overall magnetoelectrical signal has a significant angular-dependent magnon contribution, due to varying magnetostatic interactions throughout a $3 \mathrm{D}$ circuit which are not present in a standard planar magnetic device.

The insights into the influence of a $3 \mathrm{D}$ geometry on the magnetotransport effects reported here provide the basis for exploring complex spintronic effects emerging in three dimensions $^{29,30,65}$ and long-term, the realization of $3 \mathrm{D}$ devices. The methodology shown here combining FEBID 3D printing with standard planar lithography can be extended to more complex 3D geometries and other materials, leading to the fundamental study of phenomena that exploit the interplay between 3D geometry and magnetotransport. 3D spintronic effects may find key applications in the future of areas such as magnetic computing based on nanomagnetic logic, ${ }^{15,46}$ domain wall $^{14,49}$ and skyrmion devices, ${ }^{66}$ magnonics, ${ }^{29,48,67}$ magnetic neural networks, ${ }^{12}$ and frustrated magnetic systems such as artificial spin-ice systems. ${ }^{21,68,69}$

\section{METHODS}

Fabrication. The electrical contacts on which the 3D nanobridge was printed were patterned by electron-beam lithography followed by electron-beam evaporation of $5 \mathrm{~nm} \mathrm{Cr} / 50 \mathrm{~nm} \mathrm{Au}$. The milling of the trenches was performed using a Xenon Plasma focused ion beam microscope using $74 \mathrm{pA}$ current and $30 \mathrm{kV}$ acceleration voltage. The $3 \mathrm{D}$ nanostructure was printed by the same microscope using $340 \mathrm{pA}$ current, $30 \mathrm{kV}$ acceleration voltage, and $\mathrm{CO}_{2}(\mathrm{CO})_{8}$ as the precursor. The dwell points and dwell time were calculated using the algorithm developed by Skoric et al. ${ }^{53}$

Measurements. The magnetotransport measurements were performed in a bath flow Helium cryostat at a constant temperature of $180 \mathrm{~K}$. The standard four-terminal AC lock-in technique employed a constant amplitude current input of $0.6 \mu \mathrm{A}$ at a frequency of $33 \mathrm{~Hz}$.

FEM Calculations of MR. The influence of different MT effects is summarized as a magnetization-dependent resistivity tensor $\rho(m, B)$ which is reformulated from eq 1 as $\boldsymbol{E}=\boldsymbol{\rho}(\boldsymbol{m}, \boldsymbol{B}) \boldsymbol{J}$ 


$$
\boldsymbol{\rho}(\mathbf{m}, \mathbf{B})=\left(\begin{array}{ccc}
\rho_{\perp}+\Delta \rho_{m m r}+\rho_{\mathrm{G}} m_{\mathrm{x}}{ }^{2} & \rho_{\mathrm{G}} m_{\mathrm{x}} m_{\mathrm{y}}-\rho_{\mathrm{AHE}} m_{\mathrm{z}}-R_{\mathrm{OHE}} B_{z} & \rho_{\mathrm{G}} m_{\mathrm{x}} m_{\mathrm{z}}+\rho_{\mathrm{AHE}} m_{\mathrm{y}}+R_{\mathrm{OHE}} B_{y} \\
\rho_{\mathrm{G}} m_{\mathrm{x}} m_{\mathrm{y}}+\rho_{\mathrm{AHE}} m_{z}+R_{\mathrm{OHE}} B_{z} & \rho_{\perp}+\Delta \rho_{m m r}+\rho_{\mathrm{G}} m_{\mathrm{y}}{ }^{2} & \rho_{\mathrm{G}} m_{\mathrm{y}} m_{\mathrm{z}}-\rho_{\mathrm{AHE}} m_{\mathrm{x}}-R_{\mathrm{OHE}} B_{x} \\
\rho_{\mathrm{G}} m_{\mathrm{x}} m_{\mathrm{z}}-\rho_{\mathrm{AHE}} m_{\mathrm{y}}-R_{\mathrm{OHE}} B_{y} & \rho_{\mathrm{G}} m_{\mathrm{y}} m_{\mathrm{z}}+\rho_{\mathrm{AHE}} m_{\mathrm{x}}+R_{\mathrm{OHE}} B_{x} & \rho_{\perp}+\Delta \rho_{m m r}+\rho_{\mathrm{G}} m_{\mathrm{z}}{ }^{2}
\end{array}\right)
$$

where $m_{x}, m_{y}$, and $m_{z}$ are the $x, y$, and $z$ components of the magnetization vector $\boldsymbol{m}, B_{x}, B_{y}$, and $B_{z}$ are the $x, y$, and $z$ components of the total magnetic induction $\boldsymbol{B}$ and $\Delta \rho_{\mathrm{mmr}}$ is the change of resistivity due to the MMR effect.

The conductivity tensor is then obtained by inverting the resistivity tensor, $\boldsymbol{\sigma}=\boldsymbol{\rho}^{-1}$. For each section, and each field angle $\theta$, a different conductivity tensor is calculated from the modeled magnetization distribution $\boldsymbol{m}(\theta)$ and assigned to the corresponding sections of the nanobridge. The electric potential $u$ at the side contacts is then computed by solving the partial differential equation, $\nabla \cdot[-\sigma(\nabla u)]=$ 0 , using a finite element method implemented with a CAD-designbased finite element mesh that reproduces the dimensions of the printed nanobridge as shown in Figure 3a. More information on the setup of the FEM simulation is given in the SI.

\section{ASSOCIATED CONTENT}

\section{(3) Supporting Information}

The Supporting Information is available free of charge at https://pubs.acs.org/doi/10.1021/acsnano.0c10272.

Experimental and technical details (PDF)

\section{AUTHOR INFORMATION}

\section{Corresponding Authors}

Claire Donnelly - Cavendish Laboratory, University of Cambridge, Cambridge CB3 OHE, U.K.; ○ orcid.org/00000002-9942-2419; Email: cd691@cam.ac.uk

Amalio Fernández-Pacheco - Cavendish Laboratory, University of Cambridge, Cambridge CB3 OHE, U.K.; SUPA, School of Physics and Astronomy, University of Glasgow, Glasgow G12 8QQ U.K.; (1) orcid.org/0000-0002-38628472; Email: amalio.fernandez-pacheco@glasgow.ac.uk

\section{Authors}

Fanfan Meng - Cavendish Laboratory, University of Cambridge, Cambridge CB3 OHE, U.K.

Claas Abert - Faculty of Physics and Research Platform MMM Mathematics-Magnetism-Materials, University of Vienna, Vienna 1090, Austria

Luka Skoric - Cavendish Laboratory, University of Cambridge, Cambridge CB3 OHE, U.K.

Stuart Holmes - London Centre for Nanotechnology, UCL, London WC1H OAH, U.K.

Zhuocong Xiao - Nanoscience Centre, University of Cambridge, Cambridge CB3 OFF, U.K.

Jung-Wei Liao - Cavendish Laboratory, University of Cambridge, Cambridge CB3 OHE, U.K.

Peter J. Newton - Cavendish Laboratory, University of Cambridge, Cambridge CB3 OHE, U.K.

Crispin H.W. Barnes - Cavendish Laboratory, University of Cambridge, Cambridge CB3 OHE, U.K.

Dédalo Sanz-Hernández - Cavendish Laboratory, University of Cambridge, Cambridge CB3 OHE, U.K.; Unité Mixte de Physique, CNRS, Thales, Université Paris-Saclay, Palaiseau 91767, France; (1) orcid.org/0000-0002-5552-8836

Aurelio Hierro-Rodriguez - Depto. Física, Universidad de Oviedo, Oviedo 33007, Spain; SUPA, School of Physics and
Astronomy, University of Glasgow, Glasgow G12 8QQ, U.K.; ○ orcid.org/0000-0001-6600-7801

Dieter Suess - Faculty of Physics and Research Platform MMM Mathematics-Magnetism-Materials, University of Vienna, Vienna 1090, Austria

Russell P. Cowburn - Cavendish Laboratory, University of Cambridge, Cambridge CB3 OHE, U.K.

Complete contact information is available at:

https://pubs.acs.org/10.1021/acsnano.0c10272

\section{Author Contributions}

F.M., C.D., and A.F.P. designed the experiments, analyzed and interpreted the data, conceived the magnetotransport modeling methodology and wrote the manuscript. F.M, Z.X., J.W., and L.S. fabricated the samples. F.M., S.H., and P.N. performed the magnetoelectrical experiments. C.A. performed finite element micromagnetic simulations. All authors discussed the results and contributed to the final manuscript.

\section{Notes}

The authors declare no competing financial interest.

\section{ACKNOWLEDGMENTS}

This work was funded by EPSRC Early Career Fellowship EP/ M008517/1 and the Winton Program for the Physics of Sustainability. F.M. was supported by China Scholarship Council. C.D. was supported by the Leverhulme Trust (ECF-2018-016), the Isaac Newton Trust (18-08), and the L'Oréal-UNESCO U.K. and Ireland Fellowship For Women In Science. L.S. was supported by the EPSRC Cambridge NanoDTC EP/L015978/1. A.H.-R. acknowledges the support from European Union's Horizon 2020 research and innovation program under Marie Skłodowska-Curie grant ref H2020MSCA-IF-2016-746958 and from Spanish AEI under project reference PID2019-104604RB/AEI/10.13039/ 501100011033. We are grateful to Oksana ChubykaloFesenko, Can Avci and Eduardo Martínez for fruitful discussions and Sam McFadzean and William Smith for electron microscopy technical support. We note that the preprint of our manuscript is on arXiv at Meng, F.; Donnelly, C.; Abert, C.; Skoric, L.; Holmes, S.; Xiao, Z.; Liao, J.; Newton, P.; Barnes, C.; Sanz-Hernández, D.; Hierro-Rodriguez, A.; Suess, D.; Cowburn, R.; Fernández-Pacheco, A. Non-Planar Geometrical Effects on the Magnetoelectrical Signal in a Three-Dimensional Nanomagnetic Circuit. 2020, 2011.09199, arXiv. https://arxiv.org/abs/2011.09199 (Nov 18, 2020)

\section{REFERENCES}

(1) Thomson, W. XIX. On the Electro-Dynamic Qualities of Metals: Effects of Magnetization on the Electric Conductivity of Nickel and of Iron. Proc. R. Soc. London 1857, 8, 546-550.

(2) Hirohata, A.; Yamada, K.; Nakatani, Y.; Prejbeanu, L.; Diény, B.; Pirro, P.; Hillebrands, B. Review on Spintronics: Principles and Device Applications. J. Magn. Magn. Mater. 2020, 509, 166711. 
(3) Campbell, I. A.; Fert, A. Transport Properties of Ferromagnets. In Handbook of Ferromagnetic Materials; Wolforth, E. P., Ed.; NorthHolland: Amsterdam, 1982; Vol. 3, pp 747-804.

(4) Nagaosa, N.; Sinova, J.; Onoda, S.; MacDonald, A. H.; Ong, N. P. Anomalous Hall Effect. Rev. Mod. Phys. 2010, 82, 1539-1592.

(5) Baibich, M. N.; Broto, J. M.; Fert, A.; Dau, F. N. V.; Petroff, F. Giant Magnetoresistance of (001) Fe/(001)Cr Magnetic Superlattices. Phys. Rev. Lett. 1988, 61, 2472-2475.

(6) Parkin, S. S. P.; Kaiser, C.; Panchula, A.; Rice, P. M.; Hughes, B.; Samant, M.; Yang, S.-H. Giant Tunnelling Magnetoresistance at Room Temperature with $\mathrm{MgO}$ (100) Tunnel Barriers. Nat. Mater. 2004, 3, 862-867.

(7) Slonczewski, J. C. Current-Driven Excitation of Magnetic Multilayers. J. Magn. Magn. Mater. 1996, 159, L1-L7.

(8) Dieny, B.; Prejbeanu, I. L.; Garello, K.; Gambardella, P.; Freitas, P.; Lehndorff, R.; Raberg, W.; Ebels, U.; Demokritov, S. O.; Akerman, J.; Deac, A.; Pirro, P.; Adelmann, C.; Anane, A.; Chumak, A. V.; Hirohata, A.; Mangin, S.; Valenzuela, S. O.; Onbaşl1, M. C.; d'Aquino.; et al. Opportunities and Challenges for Spintronics in the Microelectronics Industry. Nat. Electron. 2020, 3, 446-459.

(9) Miron, I. M.; Garello, K.; Gaudin, G.; Zermatten, P.-J.; Costache, M. V.; Auffret, S.; Bandiera, S.; Rodmacq, B.; Schuhl, A.; Gambardella, P. Perpendicular Switching of a Single Ferromagnetic Layer Induced by In-Plane Current Injection. Nature 2011, 476, 189193.

(10) Matsukura, F.; Tokura, Y.; Ohno, H. Control of Magnetism by Electric Fields. Nat. Nanotechnol. 2015, 10, 209-220.

(11) Pan, L.; Bogy, D. B. Heat-Assisted Magnetic Recording. Nat. Photonics 2009, 3, 189-190.

(12) Torrejon, J.; Riou, M.; Araujo, F. A.; Tsunegi, S.; Khalsa, G.; Querlioz, D.; Bortolotti, P.; Cros, V.; Yakushiji, K.; Fukushima, A.; Kubota, H.; Yuasa, S.; Stiles, M. D.; Grollier, J. Neuromorphic Computing with Nanoscale Spintronic Oscillators. Nature 2017, 547, $428-431$.

(13) Fernández-Pacheco, A.; Streubel, R.; Fruchart, O.; Hertel, R.; Fischer, P.; Cowburn, R. P. Three-Dimensional Nanomagnetism. Nat. Commun. 2017, 8, 15756.

(14) Parkin, S. S. P.; Hayashi, M.; Thomas, L. Magnetic DomainWall Racetrack Memory. Science 2008, 320, 190-194.

(15) Lavrijsen, R.; Lee, J.-H.; Fernández-Pacheco, A.; Petit, D. C. M. C.; Mansell, R.; Cowburn, R. P. Magnetic Ratchet for ThreeDimensional Spintronic Memory and Logic. Nature 2013, 493, 647650.

(16) Diaz-Alvarez, A.; Higuchi, R.; Sanz-Leon, P.; Marcus, I.; Shingaya, Y.; Stieg, A. Z.; Gimzewski, J. K.; Kuncic, Z.; Nakayama, T. Emergent Dynamics of Neuromorphic Nanowire Networks. Sci. Rep. 2019, 9, 14920 .

(17) Burks, E. C.; Gilbert, D. A.; Murray, P. D.; Flores, C.; Felter, T. E.; Charnvanichborikarn, S.; Kucheyev, S. O.; Colvin, J. D.; Yin, G.; Liu, K. 3D Nanomagnetism in Low Density Interconnected Nanowire Networks. Nano Lett. 2021, 21, 716-722.

(18) Schöbitz, M.; Riz, A. D.; Martin, S.; Bochmann, S.; Thirion, C.; Vogel, J.; Foerster, M.; Aballe, L.; Menteş, T. O.; Locatelli, A.; Genuzio, F.; Le-Denmat, S.; Cagnon, L.; Toussaint, J. C.; Gusakova, D.; Bachmann, J.; Fruchart, O. Fast Domain Wall Motion Governed by Topology and CErsted Fields in Cylindrical Magnetic Nanowires. Phys. Rev. Lett. 2019, 123, 217201.

(19) Sanz-Hernández, D.; Hamans, R. F.; Liao, J.-W.; Welbourne, A.; Lavrijsen, R.; Fernández-Pacheco, A. Fabrication, Detection, and Operation of a Three-Dimensional Nanomagnetic Conduit. ACS Nano 2017, 11, 11066-11073.

(20) Perrin, Y.; Canals, B.; Rougemaille, N. Extensive Degeneracy, Coulomb Phase and Magnetic Monopoles in Artificial Square Ice. Nature 2016, 540, 410-413.

(21) May, A.; Hunt, M.; Berg, A. V. D.; Hejazi, A.; Ladak, S. Realisation of a Frustrated 3D Magnetic Nanowire Lattice. Commun. Phys. 2019, 2, 13.

(22) Jaafar, M.; Pablo-Navarro, J.; Berganza, E.; Ares, P.; Magén, C.; Masseboeuf, A.; Gatel, C.; Snoeck, E.; Gómez-Herrero, J.; Teresa, J.
M. de; Asenjo, A. Customized MFM Probes Based on Magnetic Nanorods. Nanoscale 2020, 12, 10090-10097.

(23) Ernst, D.; Melzer, M.; Makarov, D.; Bahr, F.; Hofmann, W.; Schmidt, O. G.; Zerna, T. Packaging Technologies for (Ultra-)Thin Sensor Applications in Active Magnetic Bearings. Proceedings of the 37th Int. Spring Seminar Electron Technology, Dresden, 7-11 May 2014, IEEE: Dresden, 2014, 125-129.

(24) Streubel, R.; Fischer, P.; Kronast, F.; Kravchuk, V. P.; Sheka, D. D.; Gaididei, Y.; Schmidt, O. G.; Makarov, D. Magnetism in Curved Geometries. J. Phys. D: Appl. Phys. 2016, 49, 363001.

(25) Col, S. D.; Jamet, S.; Rougemaille, N.; Locatelli, A.; Mentes, T. O.; Burgos, B. S.; Afid, R.; Darques, M.; Cagnon, L.; Toussaint, J. C.; Fruchart, O. Observation of Bloch-Point Domain Walls in Cylindrical Magnetic Nanowires. Phys. Rev. B: Condens. Matter Mater. Phys. 2014, $89,180405$.

(26) Streubel, R.; Han, L.; Kronast, F.; Ünal, A. A.; Schmidt, O. G.; Makarov, D. Imaging of Buried 3D Magnetic Rolled-Up Nanomembranes. Nano Lett. 2014, 14, 3981-3986.

(27) Hertel, R. Curvature-Induced Magnetochirality. SPIN 2013, 03, 1340009.

(28) Gaididei, Y.; Kravchuk, V. P.; Sheka, D. D. Curvature Effects in Thin Magnetic Shells. Phys. Rev. Lett. 2014, 112, 257203.

(29) Maurenbrecher, H.; Mendil, J.; Chatzipirpiridis, G.; Mattmann, M.; Pané, S.; Nelson, B. J.; Gambardella, P. Chiral Anisotropic Magnetoresistance of Ferromagnetic Helices. Appl. Phys. Lett. 2018, 112, 242401.

(30) Rikken, G. L. J. A.; Fölling, J.; Wyder, P. Electrical Magnetochiral Anisotropy. Phys. Rev. Lett. 2001, 87, 236602.

(31) Balhorn, F.; Mansfeld, S.; Krohn, A.; Topp, J.; Hansen, W.; Heitmann, D.; Mendach, S. Spin-Wave Interference in ThreeDimensional Rolled-Up Ferromagnetic Microtubes. Phys. Rev. Lett. 2010, 104, 037205.

(32) Nikonov, D. E.; Manipatruni, S.; Young, I. A. Automotion of Domain Walls for Spintronic Interconnects. J. Appl. Phys. 2014, 115, 213902.

(33) Streubel, R.; Kravchuk, V. P.; Sheka, D. D.; Makarov, D.; Kronast, F.; Schmidt, O. G.; Gaididei, Y. Equilibrium Magnetic States in Individual Hemispherical Permalloy Caps. Appl. Phys. Lett. 2012, $101,132419$.

(34) Tottori, S.; Zhang, L.; Qiu, F.; Krawczyk, K. K.; FrancoObregón, A.; Nelson, B. J. Magnetic Helical Micromachines: Fabrication, Controlled Swimming, and Cargo Transport. Adv. Mater. 2012, 24, 811-816.

(35) Albrecht, M.; Hu, G.; Guhr, I. L.; Ulbrich, T. C.; Boneberg, J.; Leiderer, P.; Schatz, G. Magnetic Multilayers on Nanospheres. Nat. Mater. 2005, 4, 203-206.

(36) Gibbs, J. G.; Mark, A. G.; Lee, T.-C.; Eslami, S.; Schamel, D.; Fischer, P. Nanohelices by Shadow Growth. Nanoscale 2014, 6, 9457-9466.

(37) Reyes, D.; Biziere, N.; Warot-Fonrose, B.; Wade, T.; Gatel, C. Magnetic Configurations in $\mathrm{Co} / \mathrm{Cu}$ Multilayered Nanowires: Evidence of Structural and Magnetic Interplay. Nano Lett. 2016, 16, $1230-1236$

(38) Kimling, J.; Kronast, F.; Martens, S.; Böhnert, T.; Martens, M.; Herrero-Albillos, J.; Tati-Bismaths, L.; Merkt, U.; Nielsch, K.; Meier, G. Photoemission Electron Microscopy of Three-Dimensional Magnetization Configurations in Core-Shell Nanostructures. Phys. Rev. B: Condens. Matter Mater. Phys. 2011, 84, 174406.

(39) Yu, X. Z.; Onose, Y.; Kanazawa, N.; Park, J. H.; Han, J. H.; Matsui, Y.; Nagaosa, N.; Tokura, Y. Real-Space Observation of a TwoDimensional Skyrmion Crystal. Nature 2010, 465, 901-904.

(40) Donnelly, C.; Finizio, S.; Gliga, S.; Holler, M.; Hrabec, A.; Odstrčil, M.; Mayr, S.; Scagnoli, V.; Heyderman, L. J.; Guizar-Sicairos, M.; Raabe, J. Time-Resolved Imaging of Three-Dimensional Nanoscale Magnetization Dynamics. Nat. Nanotechnol. 2020, 15, 356-360.

(41) Donnelly, C.; Guizar-Sicairos, M.; Scagnoli, V.; Gliga, S.; Holler, M.; Raabe, J.; Heyderman, L. J. Three-Dimensional Magnetization Structures Revealed with X-Ray Vector Nanotomography. Nature 2017, 547, 328. 
(42) Donnelly, C.; Guizar-Sicairos, M.; Scagnoli, V.; Holler, M.; Huthwelker, T.; Menzel, A.; Vartiainen, I.; Müller, E.; Kirk, E.; Gliga, S.; Raabe, J.; Heyderman, L. J. Element-Specific X-Ray Phase Tomography of 3D Structures at the Nanoscale. Phys. Rev. Lett. 2015, 114, 115501 .

(43) Fischer, P.; Sanz-Hernández, D.; Streubel, R.; FernándezPacheco, A. Launching a New Dimension with 3D Magnetic Nanostructures. APL Mater. 2020, 8, 010701.

(44) Balhorn, F.; Jeni, S.; Hansen, W.; Heitmann, D.; Mendach, S. Axial and Azimuthal Spin-Wave Eigenmodes in Rolled-Up Permalloy Stripes. Appl. Phys. Lett. 2012, 100, 222402.

(45) Sanz-Hernández, D.; Hierro-Rodriguez, A.; Donnelly, C.; Pablo-Navarro, J.; Sorrentino, A.; Pereiro, E.; Magén, C.; McVitie, S.; Teresa, J. M. de; Ferrer, S.; Fischer, P.; Fernández-Pacheco, A. Artificial Double-Helix for Geometrical Control of Magnetic Chirality. ACS Nano 2020, 14, 8084-8092.

(46) Eichwald, I.; Breitkreutz, S.; Ziemys, G.; Csaba, G.; Porod, W.; Becherer, M. Majority Logic Gate for 3D Magnetic Computing. Nanotechnology 2014, 25, 335202.

(47) Ono, T.; Miyajima, H.; Shigeto, K.; Mibu, K.; Hosoito, N.; Shinjo, T. Propagation of a Magnetic Domain Wall in a Submicrometer Magnetic Wire. Science 1999, 284, 468-470.

(48) Chumak, A. V.; Vasyuchka, V. I.; Serga, A. A.; Hillebrands, B. Magnon Spintronics. Nat. Phys. 2015, 11, 453-461.

(49) Allwood, D. A.; Xiong, G.; Faulkner, C. C.; Atkinson, D.; Petit, D.; Cowburn, R. P. Magnetic Domain-Wall Logic. Science 2005, 309, $1688-1692$.

(50) Utke, I.; Hoffmann, P.; Melngailis, J. Gas-Assisted Focused Electron Beam and Ion Beam Processing and Fabrication. J. Vac. Sci. Technology B Microelectron Nanometer. Struct. 2008, 26, 1197.

(51) Hirt, L.; Reiser, A.; Spolenak, R.; Zambelli, T. Additive Manufacturing of Metal Structures at the Micrometer Scale. Adv. Mater. 2017, 29, 1604211.

(52) Huth, M.; Porrati, F.; Schwalb, C.; Winhold, M.; Sachser, R.; Dukic, M.; Adams, J.; Fantner, G. Focused Electron Beam Induced Deposition: A Perspective. Beilstein J. Nanotechnol. 2012, 3, 597-619.

(53) Skoric, L.; Sanz-Hernández, D.; Meng, F.; Donnelly, C.; Merino-Aceituno, S.; Fernández-Pacheco, A. Layer-by-Layer Growth of Complex-Shaped Three-Dimensional Nanostructures with Focused Electron Beams. Nano Lett. 2020, 20, 184-191.

(54) Fernández-Pacheco, A.; Teresa, J. M. D.; Córdoba, R.; Ibarra, M. R. Magnetotransport Properties of High-Quality Cobalt Nanowires Grown by Focused-Electron-Beam-Induced Deposition. J. Phys. D: Appl. Phys. 2009, 42, 055005.

(55) Teresa, J. M. D.; Fernández-Pacheco, A.; Córdoba, R.; SerranoRamón, L.; Sangiao, S.; Ibarra, M. R. Review of Magnetic Nanostructures Grown by Focused Electron Beam Induced Deposition (FEBID). J. Phys. D: Appl. Phys. 2016, 49, 243003.

(56) Eden, N.; Kopnov, G.; Fraenkel, S.; Goldstein, M.; Gerber, A. Longitudinal and Transverse Magnetoresistance in Films with Tilted Out-of-Plane Magnetic Anisotropy. Phys. Rev. B: Condens. Matter Mater. Phys. 2019, 99, 064432.

(57) Smit, J. Magnetoresistance of Ferromagnetic Metals and Alloys at Low Temperatures. Physica 1951, 17, 612-627.

(58) Raquet, B.; Viret, M.; Sondergard, E.; Cespedes, O.; Mamy, R. Electron-Magnon Scattering and Magnetic Resistivity in 3D Ferromagnets. Phys. Rev. B: Condens. Matter Mater. Phys. 2002, 66, 024433.

(59) Liu, K.; Chien, C. L. Magnetic and Magneto-Transport Properties of Novel Nanostructured Networks. IEEE Trans. Magn. 1998, 34, 1021-1023.

(60) Gil, W.; Görlitz, D.; Horisberger, M.; Kötzler, J. Magnetoresistance Anisotropy of Polycrystalline Cobalt Films: GeometricalSize and Domain Effects. Phys. Rev. B: Condens. Matter Mater. Phys. 2005, 72, 134401.

(61) Sergelius, P.; Moreno, J. M. M.; Waleczek, M.; Böhnert, T.; Görlitz, D.; Nielsch, K. Magnon Contribution to the Magnetoresistance of Iron Nanowires Deposited Using Pulsed Electrodeposition. Phys. Status Solidi RRL 2015, 9, 255-258.
(62) Nguyen, V. D.; Naylor, C.; Vila, L.; Marty, A.; Laczkowski, P.; Beigné, C.; Notin, L.; Ishaque, Z.; Attané, J. P. Magnon Magnetoresistance of NiFe Nanowires: Size Dependence and Domain Wall Detection. Appl. Phys. Lett. 2011, 99, 262504.

(63) Boero, G.; Utke, I.; Bret, T.; Quack, N.; Todorova, M.; Mouaziz, S.; Kejik, P.; Brugger, J.; Popovic, R. S.; Hoffmann, P. Submicrometer Hall Devices Fabricated by Focused Electron-BeamInduced Deposition. Appl. Phys. Lett. 2005, 86, 042503.

(64) Mihai, A. P.; Attané, J. P.; Marty, A.; Warin, P.; Samson, Y. Electron-Magnon Diffusion and Magnetization Reversal Detection in FePt Thin Films. Phys. Rev. B: Condens. Matter Mater. Phys. 2008, 77, 060401.

(65) Pylypovskyi, O. V.; Sheka, D. D.; Kravchuk, V. P.; Yershov, K. V.; Makarov, D.; Gaididei, Y. Rashba Torque Driven Domain Wall Motion in Magnetic Helices. Sci. Rep. 2016, 6, 23316.

(66) Back, C.; Cros, V.; Ebert, H.; Everschor-Sitte, K.; Fert, A.; Garst, M.; Ma, T.; Mankovsky, S.; Monchesky, T. L.; Mostovoy, M.; Nagaosa, N.; Parkin, S. S. P.; Pfleiderer, C.; Reyren, N.; Rosch, A.; Taguchi, Y.; Tokura, Y.; Bergmann, K. von; Zang, J. The 2020 Skyrmionics Roadmap. J. Phys. D: Appl. Phys. 2020, 53, 363001.

(67) Woo, S.; Delaney, T.; Beach, G. S. D. Magnetic Domain Wall Depinning Assisted by Spin Wave Bursts. Nat. Phys. 2017, 13, 448454.

(68) Luo, Z.; Dao, T. P.; Hrabec, A.; Vijayakumar, J.; Kleibert, A.; Baumgartner, M.; Kirk, E.; Cui, J.; Savchenko, T.; Krishnaswamy, G.; Heyderman, L. J.; Gambardella, P. Chirally Coupled Nanomagnets. Science 2019, 363, 1435-1439.

(69) Pushp, A.; Phung, T.; Rettner, C.; Hughes, B. P.; Yang, S.-H.; Thomas, L.; Parkin, S. S. P. Domain Wall Trajectory Determined by Its Fractional Topological Edge Defects. Nat. Phys. 2013, 9, 505-511. 